\title{
COMPUTER PROCESSING OF EBIC SIGNALS
}

\author{
O.V. KONONCHUK, N.G. USHAKOV, E.B. YAKIMOV and S.I. ZAITSEV \\ Institute of Microelectronics Technology and High Purity Materials, Academy of Sciences of \\ the USSR, Chernogolovka, Moscow District 142432, USSR
}

\begin{abstract}
Some aspects of the use of computational methods are studied for quantitative EBIC measurements. It is investigated the problem of the determination of the bulk distribution of the diffusion length. Both parametric and nonparametric approaches are considered.
\end{abstract}

1.- Introduction

The electron beam induced current (EBIC) technique is widely used for the investigation of the local electrical properties of semiconductors. However the direct observation can only provide the qualitative information. To get the quantitative information one should use special methods of signal processing. There is some progress in this area recent time. Mathematical modelling has been developed by a number of authors (see for example [1-3]). There are a few examples of the solving of inverse problems of the quantitative EBIC $[4,5]$. In this paper we study some aspects of the use of numerical analysis in EBIC. We shall investigate the problem of the determination of the bulk distribution of the diffusion length using both parametric and nonparametric approaches. The problem of the estimation of results accuracy will be also considered.

\section{2. - Parametric approach}

Parametric approach is used if the investigated structure is known up to a few parameters. For example, such situation arises in the case of a single dislocation when it can be assumed as a cylinder. In this section of the paper we consider an example of the parametric estimating. It concerns to the determination of the diffusion length distribution in a silicon sample doped with gold. After reactive-ion etching gold is gettered from the subsurface region up to the depth about $10 \mu \mathrm{m}$ and its distribution, measured by DLTS, allows to present the diffusion length distribution $\mathrm{L}(\mathrm{z})$ as (3). Of course the suggested way can be used in many other situations. For simplicity we only consider the one dimensional case.

Denote by $\mathrm{G}(\mathrm{z}, \mathrm{E})$ and $\psi(z)$ the generation function and charge collection probability respectively. Then the EBIC signal I(E) can be written as 


$$
I(E)=\int_{t_{m}}^{w+t} G(z, E) d z+\int_{w+t}^{\infty} G(z, E) \psi(z) d z
$$

where $t_{m}$ is the metal thickness and $w$ is the width of the depletion region (it is assumed that plane $z=0$ coincides with the specimen surface). The charge collection probability is related with the diffusion length by the following equation (see Donolato [6]):

$$
\psi^{\prime \prime}(z)-L^{-2}(z) \psi(z)=0, \psi\left(w+t_{m}\right)=1, \lim _{z \rightarrow \infty} \psi(z)=0
$$

The main assumption about $L(z)$ is that it can be represented in the form:

$$
L^{-2}(z)=L_{0}^{-2}+L_{A u}^{-2}\left(1+B \exp \left(-\left(z-t_{m}\right) / z_{0}\right\}\right)^{-1}, z>t_{m} .
$$

The generation function $\mathrm{G}(\mathrm{z}, \mathrm{E})$ is supposed to be known (we approximated it by the Gaussian distribution).

The problem is to estimate three parameters $L_{0}, B$ and $z_{0}$ (it has been also investigated a more general case of six parameters: $\mathrm{L}_{0}, \mathrm{~L}_{A u}$, $B, z_{0}, w$ and $t_{m}$ ) and to determine confidence intervals for them. The initial data is a number of signal values observed at different values of the electron beam energy: $I\left(E_{i}\right), i=1,2, \ldots, n$. In a real experiment the signal is observed with a random error. If the error has the normal distribution and its values are independent then the most convenient way is to use the method of least squares. In the considered case it has some specific distinctions because of a complicated form of the connection between $I(E)$ and $L(z)$ (by integral equation (I) and differential equation (2)).

Denote by $\theta$ vector of the unknown parameters:

$\theta=\left(\theta_{1}, \theta_{2}, \theta_{3}\right)=\left(L_{0}, B, z_{0}\right)$. Thus the problem is to minimize

$$
\Phi(\theta)=\sum_{i=1}^{n}\left(I\left(E_{i}\right)-\int_{t_{m}}^{w+t} G\left(z, E_{i}\right) d z-\int_{w+t}^{\infty} G\left(z, E_{i}\right) \psi(z, \theta) d z\right)^{2} .
$$

The estimation can be found by means of a gradient method. On each step of the minimization it is necessary to calculate corresponding values of partial derivatives $\partial \Phi / \partial \theta_{i}, i=1,2,3$. It is easy to see that this problem is reduced to the problem of the determination of $\partial \psi / \partial \theta_{i}$. In turn $\partial \psi / \partial \theta_{i}$ can be obtained from the following equation:

$$
\begin{aligned}
\partial^{2} / \partial z^{2} & \left(\partial \psi(z, \theta) / \partial \theta_{i}\right)-L^{-2}(z)\left(\partial \psi(z, \theta) / \partial \theta_{i}\right)- \\
& -\psi(z, \theta) \partial / \partial \theta_{i}\left(L^{-2}(z)\right)=0,
\end{aligned}
$$

$\partial \psi / \partial \theta=0$ at $z=w+t_{m}$ and $\partial \psi / \partial \theta=0$ at $z+\infty$.

Thus on each step one has to solve numerically equations (2) and (4). After the estimation is found we can investigate its accuracy. Assume that errors of the signal are independent and identically distributed random variables having normal distribution with zero mean and variation $\sigma^{2}$. Let $\theta^{*}$ be the estimation obtained. Then the confidence area can be determined from the following inequality:

$$
\left(\theta-\theta^{*}\right)^{\mathrm{T}} \text { H }\left(\theta-\theta^{*}\right) / 21 \sigma^{2} \leq p
$$


where $\mathrm{p}$ is the confidence probability, $\mathrm{H}$ is the Hesse matrix: $\mathrm{H}_{\mathbf{i} j}=\partial^{2} \Phi / \partial \theta_{i} \partial \theta_{j}$ at $\theta=\theta^{*}, 1$ is the number of parameters to be estimated (in the considered case $l=3$ ), $\theta^{T}$ means $\theta$ transposed. The statistic on the left hand side of (5) has F-distribution. If $\sigma$ is unknown then it can be estimated by $s$ where

$$
s^{2}=\Phi\left(\theta^{*}\right) /(n-1)
$$

(see [7]).

3.- Determination of the diffusion length by using of the variation of the depletion region width

In order to find the bulk distribution of the diffusion length it is necessary to have a number of signals obtained under different conditions of the experiment. In other words since the unknown function $L(r)=L(x, y, z)$ depends on three variables while the signal does only on two: $x$ and $y$, it is necessary to introduce an additional parameter as the third variable. Electron beam energy is usually used as a such parameter (see $[4,5]$ ). Another way is to vary the depletion region width by means of the change of the applied voltage.

In this section we investigate some aspects of the numerical calculation of the diffusion length distribution by using of the variation of the depletion region width. We will only consider the one dimensional case because the increase of the dimension leads to a number of troubles and some of these troubles are not overcome yet. Assume that the specimen occupies the region $z<0,-\infty<x, y<\infty$, and that the diffusion length depends on $z$ but does not depend on $x$ and $y$. We also suppose for simplicity that the layer of metal is thin enough and will not take it into consideration. Denote as usual by $G(z)$ the generation
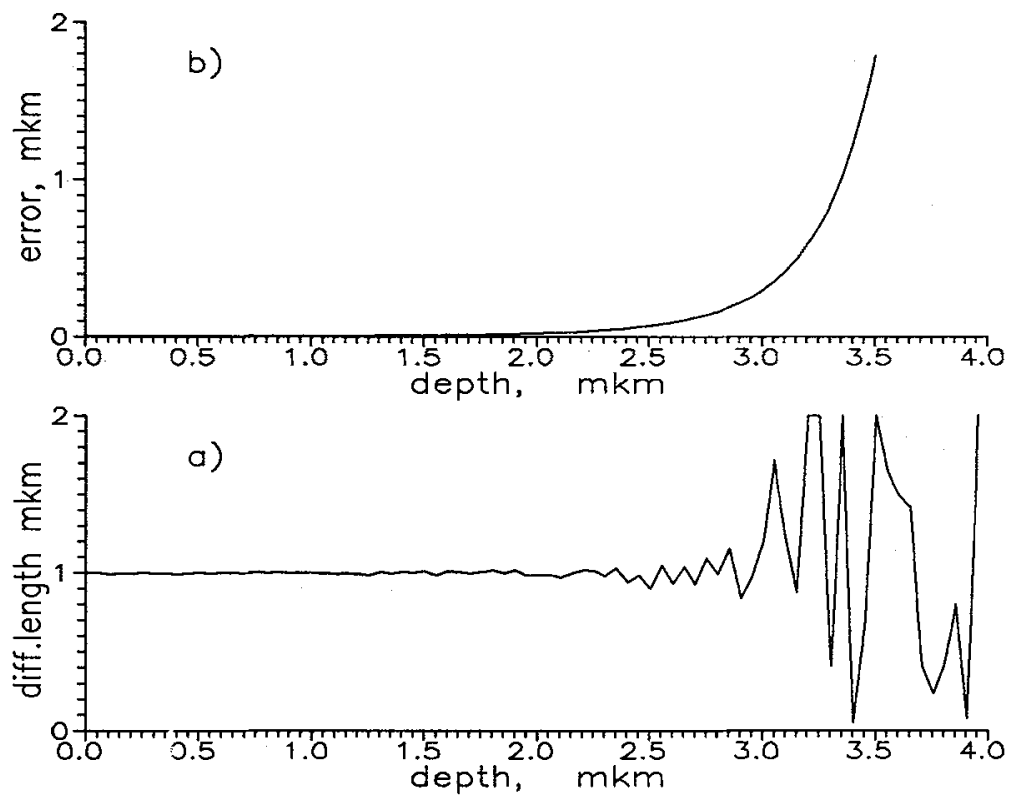

Fig. 1 - Illustration of nonstability of the problem of the diffusion length determination by variation of the depletion region. 
function and by $\psi(z, w)$ the carrier collection probability when the depletion region width is equal to $w$. Let $I(w)$ be the signal as a function of the depletion region width and $I^{\prime}(W)$ be its derivative (note that $I(w)$ and $I^{\prime}(w)$ can be measured independently). Then

$$
I(w)=\int_{-\infty}^{w} G(z) \psi(z, w) d z+\int_{w}^{0} G(z) d z .
$$

The carrier collection probability is related with the diffusion length distribution $\mathrm{L}(\mathrm{z})$ by the following equation (see above):

$$
\begin{gathered}
\partial^{2} \psi(z, w) / \partial z^{2}-L^{-2}(z) \psi(z, w)=0, z \leq w, \\
\psi(w, w)=1, \psi(-\infty, w)=0 .
\end{gathered}
$$

It is easy to see that $\psi(z, w)=\psi(z, 0) \psi^{-1}(w, 0)$. Denote $P(z)=\psi(z, 0)$. Then (6) and (7) can be written as

$$
I(w)=P^{-1}(w) \int_{-\infty}^{W} G(z) P(z) d z+\int_{w}^{0} G(z) d z
$$

and

$$
P^{\prime \prime}(z)-L^{-2}(z) P(z)=0, z<0, P(0)=1, P(-\infty)=0
$$

respectively. From $\left(6^{\prime}\right)$ we have after some simple transformations that $P^{\prime \prime}(w)=\left(Q^{2}(w)+Q^{\prime}(w)\right) P(w)$

where

$$
Q(w)=-I^{\prime}(w) /\left(I(w)-\int_{w}^{0} G(z) d z\right) .
$$

From $\left(7^{\prime}\right)$ we obtain

$$
\mathrm{L}^{2}(\mathrm{z})=\mathrm{P}(\mathrm{z}) / \mathrm{P}^{\prime \prime}(\mathrm{z})
$$

Thus

$$
L^{2}(z)=\left(Q^{2}(z)+Q^{\prime}(z)\right)^{-1}
$$

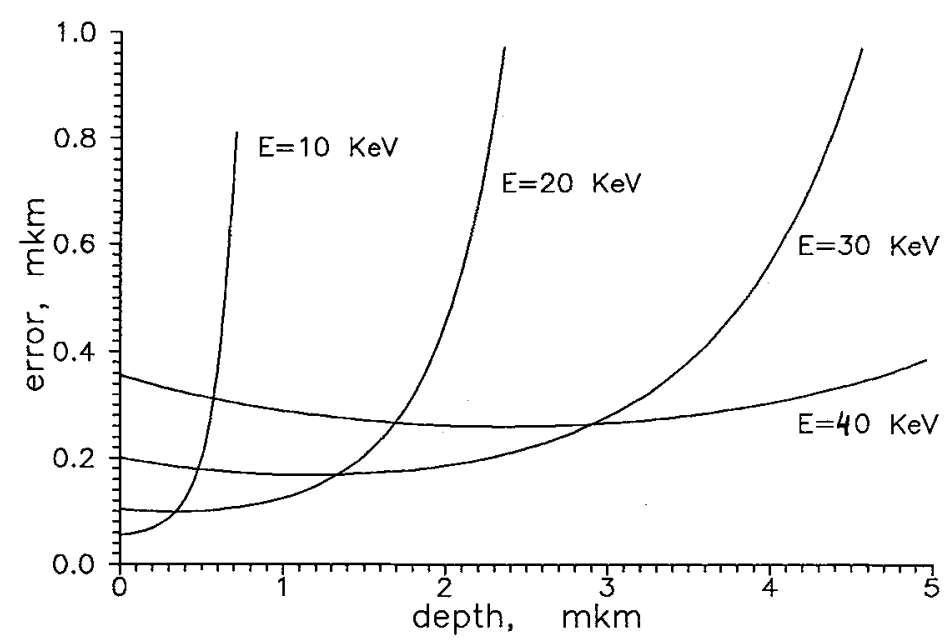

Fig. 2 - Dependence of the standard deviation of the error on the depth at different values of the beam energy. 
The problem seems to be solved. However formula (8) can be deeply nonstable with respect to errors of the signal $I(w)$ and its derivative $I^{\prime}(w)$ at some values of $w$. It is illustrated by fig la which demonstrates the diffusion length determined from (8). The true value of the diffusion length is equal to $1 \mu \mathrm{m}$ (for all $\mathrm{z}$ ). The signal obtained from (6) was contaminated by the Gaussian random noise with zero mean and standard deviation 0.001 . One can see that standard deviation of the error of the final result (the diffusion length determined from (8)) strongly depends on the depth $\mathrm{z}$ and becomes extremely big when $\mathrm{z}>3 \mu \mathrm{m}$ (fig $1 \mathrm{~b}$ shows this dependence).

The trouble can be overcome by means of the appropriate choice of the beam energy. Fig 2 demonstrates the dependence of the standard deviation of the error on the depth at different values of the beam energy (the error of the signal is the same for any curve, namely, the standard deviation of errors of $I(w)$ and $I^{\prime}(w)$ are equal to 0.02$)$. One can see, for example, that, while the diffusion length can not be determined for the depth $z=1 \mu \mathrm{m}$ at beam energy $E=10 \mathrm{KeV}$ because the error is too big it can be obtained when the beam energy equals to 20 or $30 \mathrm{KeV}$. On the other hand the error is much less at $10 \mathrm{KeV}$ when $z$ is small (less than $0.3 \mu \mathrm{m}$ ). Moreover there exists the optimal value of the beam energy for each depth. Thus for the best measurement the bias voltage and the beam energy shoud be chosen conformly.

Two examples of the determination of nonuniform distribution of the diffusion length ("step") are presented on fig 3 . One should pay to attention that the suggested method does not lead to the smoothing of the result near the "step".

\section{4.- Conclusion}

The use of numerical analysis can provide an essential increase of the effectiveness of EBIC measurements especially when one needs to obtain quantitative information. In order to have enough data for the numerical solving of a problem it is usually necessary to vary some parameter (or parameters) of the observation. Beam energy and width of the depletion region can be used as such parameters in the case of the in-
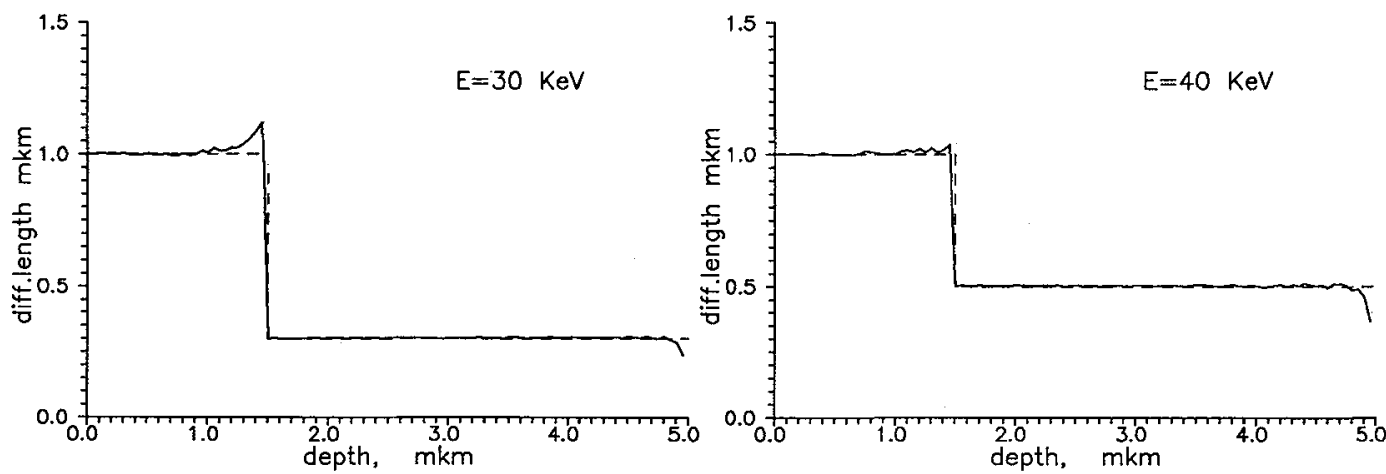

Fig. 3 - Two examples of the determination of nonuniform distribution of the diffusion length by means of the variation of the depletion region at different values of the beam energy (30 and $40 \mathrm{KeV}$ respectively). 
vestigation of the bulk distribution of electrical properties. However one should take into account that most of problems in quantitative EBIC are ill-posed. It is true even in the case of the determination of the diffusion length by means of the variation of the depletion region width although the result can be written in the form of a simple analytical formula. Sometimes such troubles can be overcome by means of choice of some parameters such as the beam energy. On the other hand it is important to use additional information. Sometimes additional information allows to formulate the problem in a parametric form what leads to much more stable computational task and hence to much more accurate result. If observed signal contains a big error then the parametric approach seems to be the only way to get meaningful result.

\section{References}

/1/ C.Donolato, optik 52 (1978) 19.

/2/ C.Donolato, J.Physique Colloq. 44 (1983) C4-269.

/3/ S.I.zaitsev and A.V.Samsonovitch, IZV. Akad. Nauk SSSR, Ser. Fiz. 51 (1987) 1587 (in russian).

/4/ S.K. Likharev, E.I.Rau, V.P.Trifonenkov and A.G.Yagola, Dokl. Akad. Nauk SSSR 307 (1989) 840 (in russian).

/5/ I.E.Bondarenko, S.K.Likharev, E.I.Rau and E.B.Yakimov, J.Crystal Growth 103 (1990) 197.

/6/ C.Donolato, J.Appl.Phys. 66 (1989) 4524.

/7/ Y.Bard, Nonlinear parameter estimation, Academic Press, 1974. 